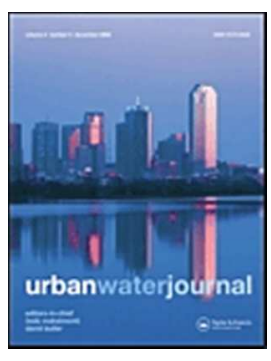

\title{
Effects of flood risk visualization format on house purchasing decisions
}

\begin{tabular}{|r|l|}
\hline Journal: & Urban Water Journal \\
\hline Manuscript ID & Draft \\
\hline Manuscript Type: & Research Article \\
\hline Date Submitted by the Author: & $\mathrm{n} / \mathrm{a}$ \\
\hline Complete List of Authors: & $\begin{array}{l}\text { Dobson, Barnaby; University of Bristol, Department of Civil Engineering } \\
\text { Miles-Wilson, Jolyon; University of Sussex, Social Decision Laboratory, } \\
\text { School of Psychology } \\
\text { Gilchrist, Iain; University of Bristol, School of Experimental Psychology } \\
\text { Leslie, David; Lancaster University, Mathematics and Statistics } \\
\text { Wagener, Thorsten; University of Bristol, Department of Civil Engineering; } \\
\text { University of Bristol, Cabot Institute }\end{array}$ \\
\hline Keywords: & Urban flooding, Flooding, Social Systems \\
\hline
\end{tabular}

\section{SCHOLARONE \\ Manuscripts}




\section{Effects of flood risk visualization format on house purchasing decisions}

2

3 Author 1

4 - Barnaby A. Dobson

5 - Department of Civil Engineering, University of Bristol, Bristol, UK

6 Author 2

7 - Jolyon J. Miles-Wilson

8 - School of Experimental Psychology, University of Bristol, Bristol, UK

9 Author 3

10 - Iain D. Gilchrist

11 - School of Experimental Psychology, University of Bristol, Bristol, UK

12 Author 4

13 - David S. Leslie

14 - Department of Mathematics and Statistics, University of Lancaster, Lancaster, UK

15 Author 5

16 - Thorsten Wagener, PhD, MSc, Dipl-Ing (FH), MICE

17 - Professor of Water and Environmental Engineering, Department of Civil Engineering,

18 University of Bristol, Bristol, UK

19 - Cabot Institute, University of Bristol, Bristol, UK

20 Full contact details of corresponding author.

21 barney.dobson@bristol.ac.uk, 07910328211, 93/95 Woodland Road, Bristol, UK, BS8 1US

224759 words in main text and table (excluding references) and 4 figures, submitted

$23 \quad 22 / 01 / 2018$ 


\section{Abstract}

26 We investigated how decision-making is affected by the visual presentation of flood risk

27 information. We exposed participants to different formats of flood risk information while

28 they simulated selecting a property to purchase. We compared three flood risk formats: (i)

29 maps currently used by the UK Environment Agency, (ii) tables that present flood level and

30 frequency information, and (iii) graphical representations depicting the level-frequency

31 combination using a cartoon house image as a physical referent. In the experiment

32 participants were presented, via computer screen, side-by-side information about two houses

33 in a series of trials. Participants made a forced choice preference judgement between 108

34 different pairs of houses to indicate which they would purchase. Our findings indicate that

35 when risk information is presented in map format, individuals are less accurate in selecting

36 lower-risk houses, compared to when the same information is presented as a graphic

37 representation of a house or as a table.

38 Keywords chosen from Urban Water Keywords

39 Urban Flooding, Flooding, Social Systems

\section{$40 \quad$ 1. Introduction}

41 Stakeholder inclusion is becoming an important factor of flood risk management. As a result,

42 data itself is insufficient and we should begin to consider its presentation and communication

43 (Newman et al., 2011). While there is increasing recognition for the necessity of public

44 consideration, quantitative investigations into how humans make decisions in the context of

45 flood risk information are still limited; flood risk communication format is a commonly

46 recommended but rarely addressed research topic (Kellens et al. 2013). A key question in

47 this context is how to present flood risk information to members of the public so that they can

48 make informed decisions. 
49 Newman et al. (2011) also state that policy change in flood prevention has partially shifted

50 responsibility for flood prevention away from the UK government. This change might be

51 driven by the realisation that resources are insufficient to fully protect everyone. This

52 assumption is reinforced by ten Brinke et al.'s (2008) suggestion that, in flood risk

53 management, the UK (as well as France and the USA) is more favourable to preparation,

54 response and recovery than to prevention (as in the Netherlands or Japan). This may be

55 because large-scale prevention projects are simply more cost effective in areas of higher

56 exposure, e.g. for regions lying below sea level. Ten Brinke et al. (2008) also discuss the

57 increasing importance of pro-action, i.e. for reducing the need for defences by avoiding high-

58 risk areas. If governments aim to move towards pro-action, with less need for prevention and

59 increased individual responsibility, then they cannot hope to do so without sufficient

60 consideration of the efficacy of communication with the public.

61 The need for good public awareness regarding flood hazards is becoming increasingly

62 evident, especially in the context of the ongoing environmental consequences associated with

63 climate change (Solomon, 2007). Researchers and governmental bodies currently emphasise

64 the necessity of publicly available flood risk information on which individuals can base

65 appropriate flood mitigation strategies. In Europe, this information is typically provided in

66 the form of flood hazard maps, as per the requirements of the 2007 Flood Directive adopted

67 by the European Parliament (Directive EU, 2007).

68 Moel et al. (2009) examined in detail the extent of flood map coverage in the EU. They

69 suggest that governmentally developed maps are created/used for emergency planning,

70 spatial planning and for raising awareness. These are predominantly frequency extent maps,

71 but occasionally flood level based ones are used (e.g. Netherlands, Germany and Beven et al.,

72 2015). Despite such maps covering large areas and often containing extensive information, it

73 appears that public awareness and appreciation of risk from flooding and its resultant 
74 preventative behaviour remains highly variable (US: Bell and Tobin, 2007: UK: Burningham

75 et al., 2008; EU: Siegrist and Heinz, 2008).

76 Many studies have provided possible explanations for this variability, such as previous

77 experience with hazards (e.g. Siegrist and Gutscher, 2006; Pagneux et al., 2011), or socio-

78 demographic variables including income (Lindell and Hwang, 2008) and home ownership

79 (Burningham et al., 2008). Although such studies have undoubtedly provided a wealth of

80 insight into the complexity of effective risk communication, implementation of their

81 contributions to risk communication media appears to be quite challenging. This perhaps lies

82 in the level of personalisation required to accommodate the variation in personality and

83 socio-demographic variables (Burningham et al., 2008). Another target for development that

84 has received relatively little attention in risk communication is the visual organisation of risk

85 communication media. There exists a considerable body of research that documents the

86 various biases of visual perception (i.e. gestalt principles) that facilitate the perception and

87 interpretation of visual scenes (e.g. Pinker, 1990; Carpenter and Shah, 1998; Kelleher and

88 Wagener, 2011), but which have been largely overlooked or underused by risk

89 communicators.

90 Bell and Tobin (2007) provide a notable exploration of different interpretations elicited

91 through different ways of presenting flood risk information. They note that flood risk

92 information is commonly based upon - and in many cases prescribed by - the 100 -year return

93 period (i.e. a 1 percent chance of a flood occurring in a given year), yet "the initial goal of

94 adopting the 100-year flood criterion was not effective communication of risk or risk policy,

95 but efficient administration and implementation" (p. 302). Indeed, the use of this criterion

96 continues to be debated (National Research Council, 2006), while it is also understandable

97 that one way of communicating cannot be equally efficient for a range of purposes. 
98 Compounding this issue is Bell and Tobin's (2007) finding that, when asked to choose what

99 aspects of flooding most concerns them - given the choices of flood water level, flooding

100 frequency, a combination of the two, or other aspects - no participant chose the frequency of

101 flooding alone as the most concerning aspect of a flood. This finding warrants concern

102 regarding the widespread use of frequency-based flood-risk communication (i.e. the 100-year

103 return period). Moreover, flood level, rather than flood frequency, emerged as the

104 predominant concern for 49 percent of participants, indicating that emphasising predicted

105 flood levels may be more relevant for public communication (a further 42 percent chose level

106 and frequency and none chose frequency alone). Developing this last point, Bell and Tobin

107 (2007) also found evidence to suggest that flood risk awareness is enhanced when physical

108 references (e.g. "the flood reaches up to the doorstep) are used to describe the extent of a

109 flood. The authors suggest, consistent with others (Smith, 2000), that including information

110 about flood extent in combination with flood frequency may prove effective. This finding

111 reinforces the availability heuristic of the importance of personal experience. Given these

112 study results, we expect that a dedicated communication method should involve a

113 combination of physical reference (i.e. flood level) and frequency.

114 Our study investigates decision-making in response to three different ways of presenting

115 flood risk information: (i) the map format currently used by the UK Environment Agency, (ii)

116 a table format that presents a matrix of flood level in combination with flood frequency, and

117 (iii) a graphical representation depicting the level-frequency combination using a cartoon

118 house image as a physical reference. The latter two formats were newly devised for this

119 experiment, drawing on the suggestions made by Bell and Tobin (2007) as discussed above.

120 Accordingly, we hypothesised that the formats utilising the combination of flood level and

121 flood frequency would lead participants to more frequently reject the higher-risk option in a

122 two-alternative forced-choice decision-making task. In our experiment, participants were 
123 asked to choose between two different houses available for sale. Details of the houses were

124 presented to participants on an information sheet that was close to the kind of sheet routinely

125 provided to home buyers (see following section). Across these sheets we manipulated how

126 the flood risk information was presented and by systematically controlling across trials all the

127 other information on the sheets we were able to measure the unique contribution the different

128 ways of presenting the flood risk information to the decision.

\section{2. Method}

\section{Experiment}

131 The study was based on examining the response of participants to different types of flood risk

132 information provided in the context of selecting a property to purchase. In the experiment

133 participants were presented on a computer screen with a series of trials in which information

134 about two houses were presented side by side (see Figure 1). Participants were asked to make

135 a forced choice preference judgment between these pairs of houses to indicate which house

136 they would consider purchasing. We manipulated the way in which the flood risk

137 information was presented (Table, Graphic or Map; See Figure 2) and the severity of the

138 flood risk (Low, Medium or High) to see how these two factors influenced the preference.

139 All participants had corrected to normal vision. Participants were selected on the basis of

140 being either previous home buyers or actively seeking to purchase a home at the time of

141 testing. All lived in or near to the city of Bristol in the UK.

142 For the map format, maps were taken from the UK Environment Agency (EA) flood risk map

143 service. They were selected from the Birmingham area of the UK. This area has a range of

144 flood risks with a relatively uniform housing style, which reduces the variability associated

145 with house style preferences. House location crosses were randomly placed in pixels that met 
146 the appropriate risk level and contained a house (this was done by RGB pixel values). It

147 should be noted that the EA does not publish precise details on how these risk levels are

148 calculated, we are simply using the risk listed in their public flood maps.

149 The remaining two display conditions in the experiment were table and graphic. In both

150 cases the flood risk information was presented in a way that decoupled flood severity and

151 recurrence interval. While the flood maps do not list depth, our study of the literature

152 suggested recipients of flood risk information find depth to be a particularly motivating

153 factor. As a result, based on correspondence with the EA, we translated these risk bands into

154 estimated depth-probability terms. These novel display methods provided the user with more

155 granular information regarding the nature of the risk for a particular property. The table

156 format aimed to mimic the European Union Energy Rating label, which is used, at least in the

157 UK, as part of the details published to advertise houses for sale. The graphic format was used

158 to give the flood risk levels a concrete perspective (Pappenberger, et al., 2013).

159 Each of the three display formats were presented at three possible levels of risk (low,

160 medium, high). The flood risk presentation format of any two pages in a comparison trial was

161 always the same. This allowed flood presentation styles to be compared without any

162 sensitivity bias from style (e.g. no comparison contained a table compared with a map). Other

163 choices were made as follows:

164 - To ensure variation could only be attributable (besides individual preferences) to

165 flood risk information only flood information on an estate agent page was altered

166 between participants. There were 3 possible risk level pairings (low/medium;

167 low/high; medium/high), 2 possible positions (right/left) and 3 formats

168 (map/table/graphic). This requires 18 participants to ensure every possible

169 combination for every possible estate agent page is viewed. 
170 - With 6 trials of every risk-position-format pairing, each participant viewed 108 trials,

$171 \quad$ thus 216 estate agent pages.

172 - Houses were paired based on number of windows, colour and size to ensure they were

173 comparable aesthetically. Besides a picture of the house; price, estate agent logo,

174 three small adverts, energy rating and search criteria were also displayed on the estate

175 agent page. These additional details were randomly assigned and fixed (across

176 participants) to that page.

177 - The picture of the house could be presented in the left or right hand side of the page.

178 The houses were selected from the suburbs of Birmingham so that they were

179 consistent with the map.

180 - House prices displayed varied randomly on a trial by trial basis between $£ 195,000$ and

$181 £ 205,000$ which was realistic for this area at the time of testing.

182 - We selected 2 different estate agent logos. Each different estate agent logo had a

183 different page layout (left aligned or right aligned) and advert associated with it.

184 - Energy ratings for houses were displayed in standard UK Energy Performance

185 Certificates format. We chose 10 pairings of Current and Potential energy rating to

186 display (Current, Potential: B,A; B,B; C,B;C,C; D,C; D,D; E,D; E,E; F,E; F,F)

187 - The search criteria were the same on every estate agent page in the experiment.

188 - Trial order was randomised for each participant.

189 - We made an a-priori decision to test 18 participants. To our knowledge there are no

190 previous studies that are similar enough to the current one to allow us to carry out a

191 formal power calculation. We selected 18 participants as this is a typical number of

192 participants testing in this broad type of behavioural experiments. As such this study

193 is exploratory. 
194

195

196

197

198

- Participants were presented with a comparison of two estate agent pages which simulated what they might see following a web search on a house purchase website. Every comparison had a different house image and flood risk level. The participant had to click on a 'buy house' button on one of the pages to proceed to the next comparison.

- Participants carried out five practice trials followed by 108 comparisons that formed the basis of the analysis reported here. At the end of the testing session we also collected and recorded post experiment feedback which is reproduced in full in the appendix.

\section{Analysis}

For the purposes of the current analysis, the response for each decision was classified as either 'correct' if the house with the lower flood risk was chosen and 'incorrect' if the house with the higher flood risk was chosen. A binary logistic regression analysis with 'correct' as the discrete dependent variable and participant, risk format, risk comparison, and a formatby-comparison interaction (e.g. map, low vs high risk) as explanatory factors was conducted to determine the effects of these variables on the probability of correct responses. 'Incorrect' was defined as the dependent reference category and participant 18, map format, and medium-high comparison were entered as the reference categories for the factors.

Binary logistic regression creates a model, based on explanatory factors, which predicts the dependent variable. For a vector of explanatory variables $x=\left(x_{1}, \ldots, x_{n}\right)$, the model fits the probability of a correct answer to be $F(x)$, satisfying

$$
\ln \left(\frac{F(x)}{1-F(x)}\right)=\beta_{0}+\beta_{1} x_{1}+\cdots+\beta_{n} x_{n}
$$


216 The coefficients, $\beta=\left(\beta_{0}, \beta_{1}, \ldots, \beta_{n}\right)$ are the unknown parameters of the model and are

217 estimated to best explain the observed data. These $\beta$ coefficients can by interpreted as the ' $\log$

218 odds ratio'; $\exp \left(\beta_{\mathrm{i}}\right)$, which indicates how much more likely the model is to produce 'correct'

219 when the explanatory factor $x_{\mathrm{i}}$ takes a value of 1 compared with when it takes a value of 0 ;

220 thus when $\beta_{\mathrm{i}}>0$ the factor $x_{\mathrm{i}}$ increases the probability of seeing the correct response, and

221 when $\beta_{\mathrm{i}}<0$ the factor $x_{\mathrm{i}}$ decreases the probability of seeing a correct response.

222 When fitting logistic regressions, the significance of a factor is assessed by a $\chi^{2}$ statistic

223 which measures the difference in the ability of the model to fit the data with or without that

224 factor present in the model. Under a null hypothesis that a factor does not enhance the ability

225 to fit the data, the $\chi^{2}$ statistic has a $\chi^{2}$ distribution with parameter equal to the number of

226 parameters added to the model (thus when the presentation format factor is added the $\chi^{2}$

227 statistic has a $\chi^{2}(2)$ distribution, because 2 additional $\beta$ parameters are added to the model

228 corresponding to the two non-reference levels of this factor). We thus compare the calculated

229 statistic to the distribution of the appropriate $\chi^{2}$ random variable; if the observed value is

230 extreme (indicated by a low $p$-value) then it is assessed that the factor is a significant

231 contributor to model fit.

\section{3. Results}

233 Figure 3 shows that the mean percentage correct was higher for the graphic and table

234 representations compared to the more widely used map representation. While all presentation

235 formats lead to a high percentage of correct choices, there is a large drop in performance if

236 the map presentation format is used. Figure 4 further shows how often the participants

237 selected correctly as a function of what risk levels the two houses were at. The graph shows

238 that the percentage correct choices was higher if the choices were between low-high and

239 between medium-high risk houses. In contrast, there was a decrease in percentage correct if 
240 one house was low risk and the other medium. This result is in line with expectations given

241 flood risk is of less importance as a decision criterion in such cases.

242 The logistic regression, as specified above, allows us to investigate which of these differences

243 are statistically significant. The outcome of this modelling exercise is shown in Table 1.

244 Critically for the current study there was a reliable effect of the presentation format $\left(\chi^{2}(2)=\right.$

$24521.12, p<0.001)$ indicating that we have strong evidence that participants' responses were

246 affected by how the information was presented. There was also a robust effect of risk

247 comparison $\left(\chi^{2}(2)=33.39, p<0.001\right)$ indicating that participants were sensitive to the

248 relative flood risk between the two houses presented in any given trial. There was no

249 evidence for a reliable interaction between these two factors $\left(\chi^{2}(4)=0.95, p=0.917\right)$. This

250 suggests that the presentation type and the risk combine in an additive manner to affect the

251 choices made. Less centrally to the focus of this paper there was also a main effects of

252 participant $\left(\chi^{2}(17)=102.01, p<0.001\right)$ indicating that there were reliable individual

253 differences between the participants in how they responded; these are among the strongest

254 effects and are account for personal differences (e.g. education or age) between participants.

255 As shown in Table 1 and Figure 3, graphic presentation $\left(\chi^{2}(1)=4.45, p=0.035\right)$, and table

256 presentation $\left(\chi^{2}(1)=7.70, p=0.006\right)$, are both significantly more likely to produce a correct

257 response than map presentation (the reference category). Low-medium comparisons $\left(\chi^{2}(1)=\right.$

$2584.43, p=0.035)$, were observed to produce significantly lower probabilities of correct

259 responses than medium-high comparisons (reference category), whilst low-high comparisons

260 were not found to differ, as also illustrated in Figure 4. Again, we found no evidence that the

261 format of presentation interacted with the risk difference to produce a greater effect of one

262 presentation mode at a particular risk level. 
263 At the end of the experiment participants were asked to provide comments about

264 their experience. In particular, participants were asked what they thought about the

265 flood-risk presentation formats and whether they adopted a particular strategy

266 throughout the experiment. Though these comments have not been analysed

267 quantitatively, a brief summary describing trends in the responses is provided below.

268 Participant comments are also provided in Appendix A.

269 In general, participants appeared to prefer the graphic and/or table presentation

270 formats over the map format. Specifically, six out of 18 participants preferred

271 graphic presentation, five of 18 preferred table format, whilst only three of 18

272 described a preference for map format. Two of those preferring maps expressed

273 confusion about frequency-based flood predictions in graphic and table

274 presentations, whilst the third found it difficult to see the different flood levels

275 presented in the graphic format. Four out of 18 participants did not express a clear

276 preference for any one format.

277 The map format was repeatedly described as causing confusion due to the extent of

278 light and dark blue depicting relative risk; a lack of defining borders and the use of

279 different hues of the same colour were reported to make certain areas appear more

280 risky than they actually were, this is consistent with the results reported by (Ratwani

$281 \&$ Trafton, 2008). Another recurrent theme in participants' comments was a feeling

282 of lack of control in dealing with flood-risk level; participants often expressed that

283 they could not "do anything" about flood-risk level or where they live, but that they

284 can "do something" about, for example, poor energy performance (e.g. install

285 insulation). Thus, poor energy performance was generally more acceptable to

286 participants than high flood-risk. 
288 The present experiment investigated how decision-making based on flood risk information is

289 affected by the way in which this information is visually presented. We compared three ways

290 of presenting the same flood risk information: (i) the map format of presentation currently

291 used by the UK Environment Agency, (ii) a table format that presents flood level information

292 in combination with flood frequency information, and (iii) a graphical representation

293 depicting the level-frequency combination using a cartoon house image as a physical referent.

294 Our findings indicate that when risk information is presented in map format, individuals are

295 less accurate in selecting lower-risk houses, compared to when the same information is

296 presented as a graphic representation of a house or as a table (Figure 1). In addition, we find

297 evidence for a reliable ability to avoid high-risk houses when they were presented jointly with

298 either low or medium risk houses (Figure 2). We find no evidence of an interaction between

299 these two effects.

300 This results pattern is consistent with previous research into presenting flood risk

301 information. For example, Bell and Tobin (2007) compared participants' responses to four

302 ways of presenting the risk of a 100-year flood event (' 100 -year flood' vs. ' 1 percent chance

303 in any given year' vs. '26 percent chance occurring in 30 years' vs. a flood risk map) and

304 found the 1 percent description to be consistently more effective in conveying uncertainty

305 than the 100-year description. Conversely, the 1 percent description was found to perform

306 worse than the 100-year description in motivating concern or protection (e.g. preventative

307 behaviour), a finding that indicates - as the present study does - that differing presentations

308 of risk elicit differing conclusions by the viewer; i.e. composition affects conclusion. This

309 result further highlights the need to consider what the intended message of a risk

310 communication medium is during its design already. 
311 In contrast to the wealth of research dedicated to investigating individual differences in flood

312 risk communication and perception, there is markedly less attention focused on how the

313 visual features of flood-risk presentation affect their interpretation, despite an extensive

314 literature on the perception of graphical representations of quantitative information (e.g.

315 (Carpenter \& Shah, 1998), (Cleveland \& McGill, 1986), (Shah \& Freedman, 2011)). For

316 example, models of visual display comprehension emphasise an interaction between top-

317 down (e.g. content familiarity, graph skills; i.e. individual differences) and bottom-up (e.g.

318 visual features of the display) processes when decoding information from visual displays

319 (Hegarty, 2005), (Kriz \& Hegarty, 2007). In the case of graph comprehension, for example,

320 Shah and Freedman (2011) showed that the same quantitative information is interpreted

321 differently when presented in bar graphs as compared to line graphs, and prior knowledge

322 (i.e. top-down processing) was found to interact with the influence of presentation format.

323 The authors suggest that these systematic differences in interpretation can in part be

324 understood in the context of Gestalt Laws of Perceptual Organisation (Wertheimer, 1938).

325 Indeed, other researchers (Pinker, 1990) have provided detailed theories of how Gestalt

326 principles such as those of similarity, proximity, and good continuity are used by viewers to

327 manage the cognitive processing demands of graphical displays (Shah, et al., 1999). In light

328 of this, it is surprising that the role of such bottom-up processing (and its interaction with top-

329 down processing) has so far been largely under-studied in the context of flood-risk

330 communication.

331 The influence of the aforementioned Gestalt principles on interpretation is evident in the

332 presentation formats used in the present experiment. The table format in particular is

333 conducive to organising the displayed information based on the good continuity provided by

334 its columns, whilst the principle of proximity is likely to facilitate flood level comparisons in

335 the graphic format. These principles influence what inferences are made by directing 
336 attention toward and facilitating the processing of particular elements of the visual display. A

337 possible explanation for the varied success of map presentations of flood-risk information

338 may thus be the absence of these perceptual elements that direct, focus, and facilitate the

339 cognitive processing of visual displays. It would therefore be useful in future studies to

340 further explore visual elements of flood-risk presentation mediums that are particularly

341 effective in encouraging appropriate inference generation.

342 We have made the assumption that the desired impact on behaviour of flood risk

343 communication is lesser acceptance of higher risks; we have treated flood communication as

344 successful if the viewer accepts the lower-risk option. This was necessary to be able to

345 measure participants' decision-making behaviour in response to the different presentation

346 formats, although we acknowledge that this may not be the desired output of flood risk

347 communication in all cases in the real world. Were this the case, there already exists evidence

348 to suggest that an effective way to do this would be to communicate affect-laden flood-risk

349 messages that induce, for example, fear of flood events (Keller, et al., 2006). However, such

350 an approach is likely to leave viewers relatively over-sensitised to risk, and in reality it is not

351 the goal of flood-risk communication to 'scare' the public into avoiding all higher-risk

352 options but rather to make a more informed decision. As such, our simplifying assumption

353 may constrain generalisation to instances in the real world, and future research would benefit

354 from the adoption of paradigms that do not treat risk-aversion per se as the desirable

355 behavioural outcome. One possibility may be to frame similar experiments in the context of

356 an economic game, where participants would have the opportunity to make a profit based on

357 the odds of their decisions; in such cases, high-risk decisions may provide greater payoffs and

358 so will not be ubiquitously avoided.

\section{5. Practical Relevance and Potential Applications}


360 The present experiment evidences an important influence of the visual format of flood-risk

361 communication mediums on viewers' interpretations. Our key finding - that participants are

362 more accepting of high probability flood risks when this information is presented in map

363 format as compared to the graphic and table format - is particularly relevant for flood-risk

364 communicators in countries that currently employ flood hazard maps as their primary method

365 for communication. For members of the European Union in particular, the finding that flood

366 hazard maps encourage greater risk acceptance than other types of (newly conceived) risk

367 presentation is potentially problematic in light of the fact that this method of communication

368 is currently prescribed by the 2007 Flood Directive of European Parliament (2007/60/EC). It

369 is possible that an over-focus on individual and socio-demographic variables and an under-

370 focus on visual presentation factors may in part explain why improving public risk awareness

371 is such a challenge. Further investigation of the influence of different formats of flood-risk

372 presentation may provide useful insight for flood-risk communicators, who may wish to

373 implement what is known about graphical representation and the influence of visual (bottom-

374 up) elements of graphical displays in addition to tackling the variation resulting from (top-

375 down) socio-demographic and individual differences. The implications of our findings add to

376 those of others who have similarly identified issues with the use of the 100 -year return period

377 (Bell \& Tobin, 2007). These early-stage findings highlight a need for greater consideration of

378 presentation format in flood-risk communication, and future research in this area is likely to

379 prove useful in improving public awareness and understanding of risk from flood events.

\section{$380 \quad$ Table 1}

381 Model parameter estimates of binary logistic regression analysis, showing parameter values

382 of beta $(\beta)$, standard error of beta $(\operatorname{SE} \beta)$, odds ratio $(\exp (\beta))$, and odds ratio confidence 
383 intervals, with 'correct' as the dependent variable and 'participant', 'presentation format', and 384 'risk comparison' as factors.

\begin{tabular}{|c|c|c|c|c|c|}
\hline \multirow[b]{2}{*}{ Included } & \multirow[b]{2}{*}{$\beta$} & \multirow[b]{2}{*}{ SE $\beta$} & \multicolumn{3}{|c|}{ 95\% Cl for Odds Ratio } \\
\hline & & & Odds ratio & Lower & Upper \\
\hline Constant & 1.99 & 0.36 & 7.31 & 3.64 & 14.69 \\
\hline \multicolumn{6}{|l|}{ Participant } \\
\hline 1 & $-1.21^{*}$ & 0.39 & 0.30 & 0.14 & 0.64 \\
\hline 2 & $-1.25^{*}$ & 0.39 & 0.29 & 0.13 & 0.61 \\
\hline 3 & -0.51 & 0.42 & 0.60 & 0.27 & 1.36 \\
\hline 4 & -0.51 & 0.42 & 0.60 & 0.27 & 1.36 \\
\hline 5 & -0.58 & 0.41 & 0.56 & 0.25 & 1.26 \\
\hline 6 & $-0.89^{*}$ & 0.40 & 0.41 & 0.19 & 0.90 \\
\hline 7 & 0.23 & 0.48 & 1.25 & 0.49 & 3.18 \\
\hline 8 & $-1.78^{* *}$ & 0.38 & 0.17 & 0.08 & 0.36 \\
\hline 9 & -0.36 & 0.43 & 0.70 & 0.30 & 1.61 \\
\hline 10 & $-1.86^{* *}$ & 0.38 & 0.16 & 0.07 & 0.33 \\
\hline 11 & 0.35 & 0.49 & 1.43 & 0.55 & 3.72 \\
\hline 12 & -0.65 & 0.41 & 0.52 & 0.23 & 1.17 \\
\hline 13 & -0.51 & 0.42 & 0.60 & 0.27 & 1.36 \\
\hline 14 & -0.77 & 0.40 & 0.46 & 0.21 & 1.02 \\
\hline 15 & -0.44 & 0.42 & 0.65 & 0.28 & 1.48 \\
\hline 16 & $-1.57^{* *}$ & 0.38 & 0.21 & 0.10 & 0.44 \\
\hline 17 & -0.71 & 0.41 & 0.49 & 0.22 & 1.09 \\
\hline $18^{\text {REF }}$ & 0 & & 1.00 & & \\
\hline \multicolumn{6}{|l|}{ Format } \\
\hline Graphic & $0.54^{*}$ & 0.25 & 1.71 & 1.04 & 2.81 \\
\hline Table & $0.73^{*}$ & 0.26 & 2.08 & 1.24 & 3.48 \\
\hline $\mathrm{Map}^{\mathrm{K} t \mathrm{r}}$ & 0 & & 1.00 & & \\
\hline Comparison & & & & & \\
\hline
\end{tabular}


Format-Comparison Interaction

$\begin{array}{llllll}\text { Graphic by Low-Medium } \quad-0.17 & 0.34 & 0.84 & 0.44 & 1.63\end{array}$

$\begin{array}{llllll}\text { Graphic by Low-High } & 0.05 & 0.37 & 1.06 & 0.51 & 2.17\end{array}$

Graphic by Medium-High ${ }^{\text {Ktr }} \quad 0$

1.00

$\begin{array}{llllll}\text { Table by Low-Medium } & -0.29 & 0.35 & 0.75 & 0.38 & 1.48\end{array}$

$\begin{array}{llllll}\text { Table by Low-High } & -0.06 & 0.38 & 0.95 & 0.45 & 1.98\end{array}$

Table by Medium-High $^{\text {Ktㅏ }} \quad 0 \quad 1.00$

Map by Low-Medium $^{\text {REF }} \quad 0 \quad 1.00$

Map by Low-High ${ }^{\text {REF }} \quad 0 \quad 1.00$

Map by Medium-High ${ }^{\text {REF }} \quad 0 \quad 1.00$

385

386 Note: Model $\chi^{2}(25)=158.46, p<.001 .{ }^{*} p<.05 .{ }^{*} p<.001$. REF $=$ Reference category.

\section{Appendix}

389 Participants' comments and opinions having completed the experiment.

\begin{tabular}{ll}
\hline Participant & Comments \\
\hline 1 & - Map presentation easiest to use. \\
& - Experience of house being flooded. \\
& - Awareness of recent flooding events. \\
& - Strategy: Compared images, followed by energy, followed by flood risk. \\
& - Graphic presentation very powerful.
\end{tabular}


- Maps difficult to understand.

- Strategy: Compared images, followed by energy, followed by flood risk, followed by price.

- Flood risk had large influence on decisions.

- Began to ignore price.

- Aesthetics of the houses also had some influence.

- Graphic presentation best.

- Began to ignore energy performance information.

- Also began to ignore flood-risk information as this participant had previous experience with unreliable flood-risk information.

- Graphic and table presentations better than map presentation.

- Map difficult to understand.

- Strategy: Checked flood risk, followed by energy performance. If energy performance for a house was high it was further considered, even if it was at high risk of flooding. Price considered with respect to what improvements could be carried out on house (e.g. build an extension).

- Graphic presentation best; easy to see flood levels.

- Table presentation second best.

- Maps hardest to use.

- Strategy: At start compared all aspects (e.g. aesthetics, price, etc.) and compared flood-risk information last. Later started to compare floodrisk information first and then compared other things. Energy performance largely ignored. Willing to pay more for a lower risk house. Tendency to initially prefer aesthetically pleasing houses, but this 
preference reduced if house was at high risk.

- Participant felt that flood risk information would be useful when buying a house; had not previously considered this when buying a house.

- Graphic presentation best.

- Map presentation difficult to understand.

- Strategy: Energy performance information ignored because one can "do something" about poor energy performance but one cannot do anything about the risk of flood. Aesthetic preference played a role, but swayed by flood-risk level.

- Flood risk information should be provided with house information when looking to buy so that a fully informed decision can be made.

- This participant independently investigated flood-risk information when buying their house.

- Table presentation best.

- Graphic presentation second best.

- Map presentation most difficult.

- Strategy: Compared aesthetics, followed by price, followed by floodrisk information. Ignored energy performance information because one can "do something" about energy performance.

- It would be useful if this information was provided on estate agent websites.

- Table presentation best.

- Graphic presentation more difficult to use.

- Strategy: Largely influenced by flood-risk information. Ignored energy 
performance information because one can "do something" about it (e.g. insulation). Ignored price.

- No data available.

- Map presentation was easiest to use.

- Other presentation formats were more difficult because of use of fractions (frequency probabilities) to describe risk.

- Graphic presentation was easiest to use.

- Map presentation was hardest to use; crosses surrounded by lots of blue make it look more risky.

- Strategy: Largely influenced by aesthetics, followed by flood-risk information.

- Graphic presentation was most informative.

- Strategy: Compared energy performance and flood risk information.

Participant noticed that these were the only factors that considerably changed.

- Table presentation easiest to use.

- Map presentation more difficult to use.

- "Fear factor" associated with picture; high flood level for a rare flood still appears off-putting.

- It would be useful for flood risk information to be provided when viewing houses.

- This participant has bought several houses in the past.

- Map presentation most difficult to use.

- Graphic and table presentations equally easy/difficult to use. 
- On first trial struggled slightly with understanding table presentation; fractions (flood frequencies) and flood level slightly confusing.

- Didn't really like the look of the houses.

- Flood risk information would be useful when looking at houses.

16

17

18

- Graphic presentation was confusing because the lowest two flood level estimates were very close together and thus difficult to see.

- Table presentation most useful.

- Strategy: As experiment progressed participant tended to forget about attending to certain factors, including flood-risk information.

- Flood-risk information would be useful to have provided when looking to buy a house. 


\section{Acknowledgements}

392 The UK Environment Agency for their advice in appropriate frequencies and levels. This

393 work was supported by the Natural Environment Research Council [Consortium on Risk in

394 the Environment: Diagnostics, Integration, Benchmarking, Learning and Elicitation

395 (CREDIBLE); grant number NE/J017450/1] and by the UK Engineering and Physical

396 Sciences Research Council [Decision making in an unstable world; grant number

$397 \mathrm{EP} / \mathrm{I} 032622 / 1]$.

\section{Bibliography}

399 Bell, H. M. \& Tobin, G. A., 2007. Efficient and effective? The 100-year flood in the

400 communication and perception of flood risk.. Environmental Hazards, 7(4), pp. 302-311.

401 Beven, K., Lamn, R., Leedal, D. \& Hunter, N., 2007. Communicating uncertainty in flood 402 inundation mapping: a case study., International Jounrla of River Basin Management, 13(3), 403 pp. 285-295.

404 Burningham, K., Fielding, J. \& Thrush, D., 2008. It'll never happen to me': understanding 405 public awareness of local flood risk.. Disasters, 32(2), pp. 216-238.

406 Carpenter, P. A. \& Shah, P., 1998. A model of the perceptual and conceptual processes in 407 graph comprehension.. Journal of Experimental Psychology: Applied, 4(2), p. 75.

408 Cleveland, W. S. \& McGill, R., 1986. An experiment in graphical perception. nternational 409 Journal of Man-Machine Studies, 25(5), pp. 491-500.

410 Directive, E. U., 2007. 60/EC of the European Parliament and of the Council of 23 October 4112007 on the assessment and management of flood risks.. Journal of the European Union L, 412 288(27). 
413 Hegarty, M., 2005. Multimedia learning about physical systems. In: The Cambridge

414 handbook of multimedia learning.. s.1.:s.n., pp. 445-465.

415 Kelleher, C., \& Wagener, T., 2011. 10 rules for effective data visualization. Environmental

416 Modeling and Software, doi:10.1016/j.envsoft.2010.12.006.

417 Keller, C., Siegrist, M. \& Gutscher, H., 2006. The role of the affect and availability heuristics 418 in risk communication. Risk Analysis, 26(3), pp. 631-639.

419 Kellens, W., Terpstra, T. \& Maeyer, P. d., 2013. Perception and communication of flood

420 risks: A systematic review of empirical research. Risk Analysis, 33(1), pp. 24-49.

421 Kriz, S. \& Hegarty, M., 2007. Top-down and bottom-up influences on learning from

422 animations.. International Journal of Human-Computer Studies, 65(11), pp. 911-930.

423 Lindell, M. K. \& Hwang, S. N., 2008. Households' perceived personal risk and responses in a 424 multihazard environment.. Risk Analysis, 28(2), pp. 539-556.

425 Moel, H. d., Alphen, J. v. \& Aerts, J. C. J. H., 2009. Flood maps in Europe-methods, 426 availability and use.. Natural Hazards and Earth System Science, 9(2), pp. 289-301.

427 National Research Council, 2006. Completing the forecast: characterizing and

428 communicating uncertainty for better decision using weather and climate forecasts. Washingt 429 DC, National Academy Press.

430 Newman, R., Ashley, R., Molyneux-Hodgson, S. \& Cashman, A., 2011. Managing water as a

431 socio-technical system: the shift from 'experts' to 'alliances'. Engineering Sustainability,

432 164(ES1), pp. 95-102. 
433 Pagneux, E., Gísladóttir, G., \& Jónsdóttir, S. (2011). Public perception of flood hazard and

434 flood risk in Iceland: a case study in a watershed prone to ice-jam floods.. Natural hazards, 435 58(1), pp. 269-287.

436 Pappenberger, F. et al., 2013. Visualizing probabilistic flood forecast infromationL: expert

437 preferences and perceptions of best practice in uncertainty communication. Hydrological

$438 \quad$ Processes, Volume 27, pp. 132-146.

439 Pinker, S., 1990. A theory of graph comprehension. Artificial intelligence and the future of 440 testing, pp. 73-126.

441 Ratwani, R. M. \& Trafton, J. G., 2008. Thinking graphically: connecting vision and cognition 442 during graph comprehension. Jounarl of Experimental Psychology: Applied, 14(1), pp. 36-49.

443 Shah, P. \& Freedman, E. G., 2011. Bar and Line Graph Comprehension: An interaction of 444 top-down and bottom-up processes.. Topics in Cognitive Science, 3(3), pp. 560-578.

445 Shah, P., Mayer, R. E. \& Hegarty, M., 1999. Graphs as aids to knowledge construction:

446 Signaling techniques for guiding the process of graph comprehension. Journal of Educational 447 Psychology, 91(4), p. 690.

448 Siegrist, M. \& Gutscher, H., 2006. Flooding risks: A comparison of lay people's perceptions

449 and expert's assessments in Switzerland.. Risk Analysis, 26(4), pp. 971-979.

450 Siegrist, M. \& Heinz, G., 2008. Natural hazards and motivation for mitigation behavior:

451 People cannot predict the affect evoked by a severe flood.. Risk Analysis, 28(3), pp. 771-778.

452 Smith, D. I., 2000. Floodplain management: problems, issues and opportunities . In: D. J.

453 Parker, ed. Floods vol. I.. London: Routledge, pp. 254-267. 
454 Solomon, S., 2007. Climate change 2007-the physical science basis: Working group I

455 contribution to the fourth assessment report of the IPCC (Vol 4.). s.1.:Cambridge University

456 Press.

457 ten Brinke, W. B., Saeijs, G. E., Helsloot, I. \& van Alphen, J., 2008. Safety chain approach in

458 flood risk management. Proceedings of the ICE-Municipal Engineer, 161(2), pp. 93-102.

459 Wertheimer, M., 1938. Laws of organization in perceptual forms.. In: A source book of

460 Gestalt psychology. London: s.n., pp. 71-88.

\section{$461 \quad$ Figures}

462 Figure 1: Example display in the experiment that shows two different real estate

463 advertisements including information on flood risk in the bottom left. Participants were

464 asked to select the house that they would prefer.

465 Figure 2: The three ways of presenting the flood risk information that were used on the real

466 estate advertisements: Table (top row); Graphic (middle row) and Map (bottom row) for the

467 three levels of risk: Low Risk (first column); Medium Risk (second column); High Risk

468 (third column).

469 Figure 3. Mean percentage of trials in which the lower risk property was selected

470 within each presentation format $(\mathrm{N}=648$ for each format; Total $\mathrm{N}=1944)$. Error

471 bars depict the standard error of the estimate of the mean.

472 Figure 4. Mean percentage of trials in which the lower risk property was selected

473 within each risk comparison $(\mathrm{N}=648$ for each comparison; Total $\mathrm{N}=1944)$. Error

474 bars depict the standard error of the mean. 

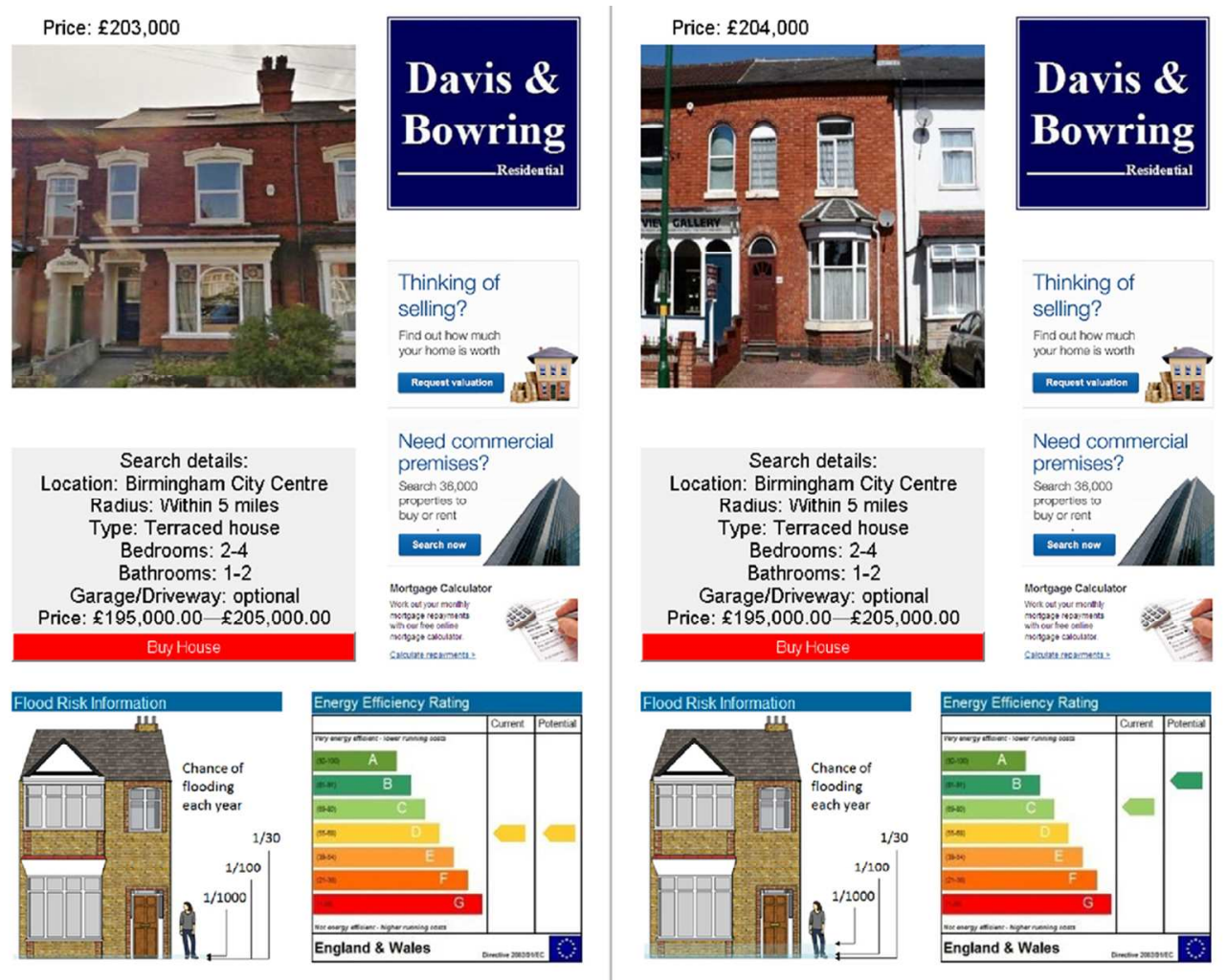
Radius: Within 5 miles Type: Terraced house Bedrooms: $2-4$

Bathrooms: $1-2$ Price: $£ 195,000.00-£ 205,000.00$

Need commercial premises?

Search 36,000
propertes to properties to
buy ar rent search now search now
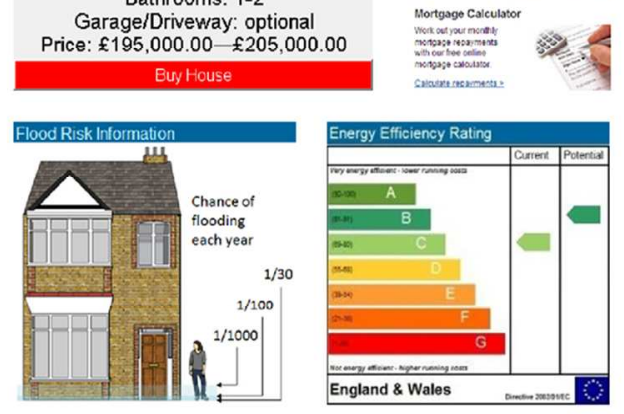

Figure 1: Example display in the experiment that shows two different real estate advertisements including information on flood risk in the bottom left. Participants were asked to select the house that they would prefer.

$419 \times 331 \mathrm{~mm}(72 \times 72 \mathrm{DPI})$ 


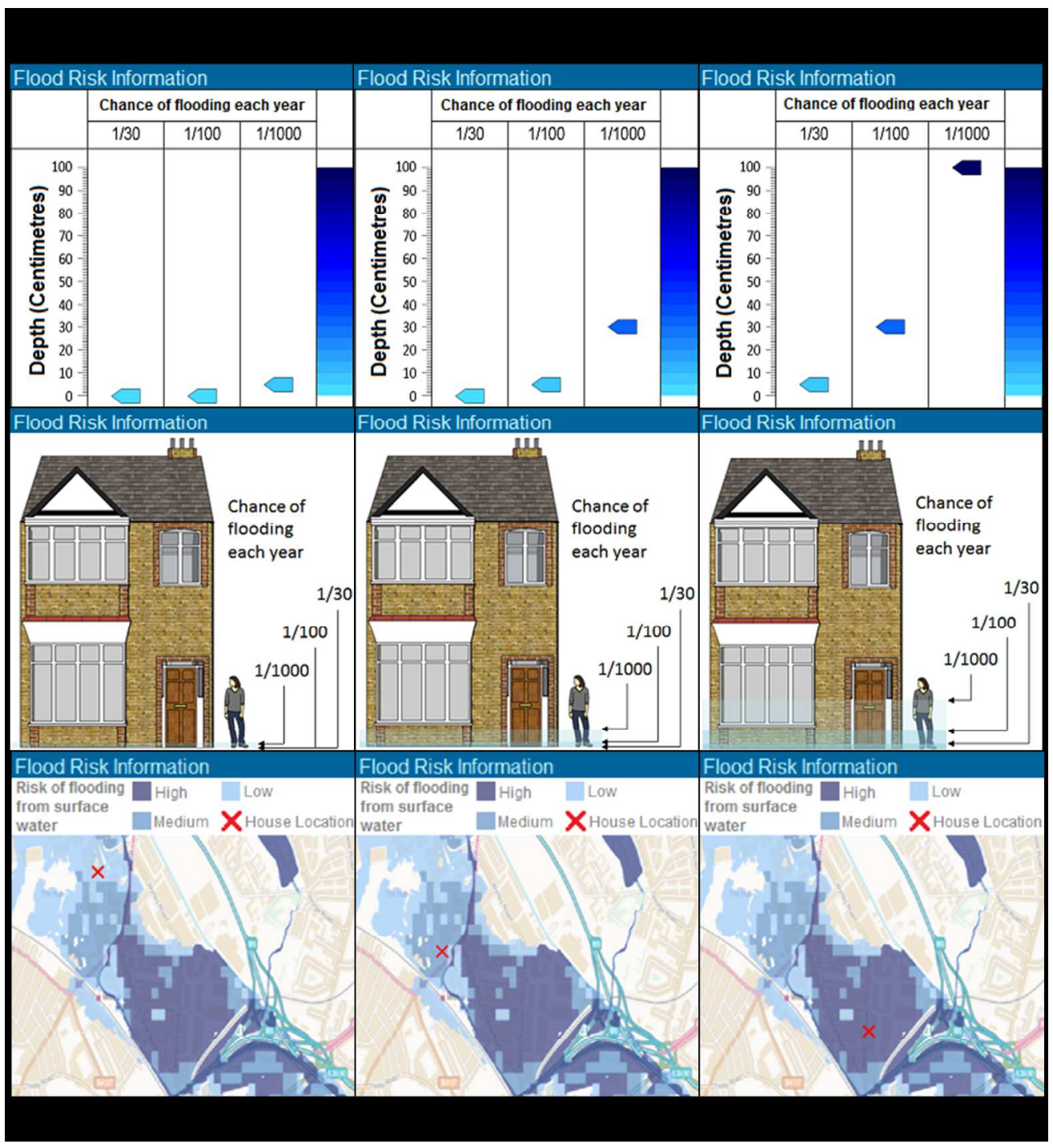

Figure 2: The three ways of presenting the flood risk information that were used on the real estate advertisements: Table (top row); Graphic (middle row) and Map (bottom row) for the three levels of risk: Low Risk (first column); Medium Risk (second column); High Risk (third column).

$1335 \times 1449 \mathrm{~mm}(72 \times 72 \mathrm{DPI})$ 


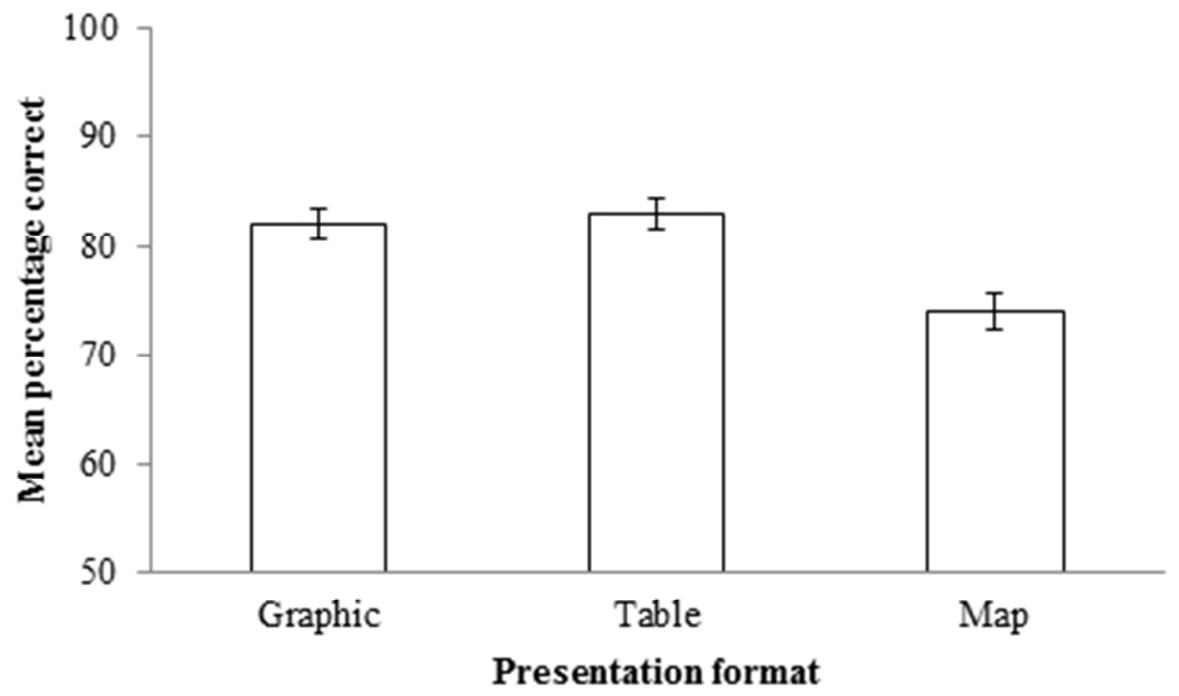

Figure 3. Mean percentage of trials in which the lower risk property was selected within each presentation format ( $\mathrm{N}=648$ for each format; Total $\mathrm{N}=1944$ ). Error bars depict the standard error of the estimate of the mean.

$178 \times 99 \mathrm{~mm}(72 \times 72 \mathrm{DPI})$ 
Figure 4. Mean percentage of trials in which the lower risk property was selected within each risk comparison $(\mathrm{N}=648$ for each comparison; Total $\mathrm{N}=1944)$. Error bars depict the standard error of the mean.

$$
169 \times 101 \mathrm{~mm}(72 \times 72 \mathrm{DPI})
$$

